\title{
O desafio do restabelecimento de um sorriso antiestético por meio de prótese fixa metal-free
}

\author{
The challenge of restoring an unsightly smile by means of a fixed metal-free prosthesis \\ El desafío del restablecimiento de una sonrisa antiestética por medio de prótesis fija metal-free \\ Caroline de Freitas JORGE \\ Sandro Basso BITENCOURT \\ Letícia Cerri MAZZA \\ Marcio CAMPANER \\ Juliana Lujan BRUNETTO \\ Letícia de Paula Gimenez BILLOBA \\ Daniela Micheline dos SANTOS \\ Aldiéris Alves PESQUEIRA \\ Departamento de Materiais Odontológicos e Prótese, Faculdade de Odontologia de Araçatuba, FOA-UNESP Univ. Estadual Paulista, \\ 16015-050 Araçatuba SP, Brasil
}

\section{Resumo}

Para alcançar a estética em dentes anteriores deve-se ter conhecimento de diversas técnicas e principalmente fazer a escolha correta dos materiais. As cerâmicas odontológicas estão cada vez mais presentes nas restaurações, visto que possuem ótimas propriedades ópticas e mecânicas. Assim, o objetivo deste estudo foi proporcionar a estética em dentes anteriores com diferentes subtratos através de uma prótese fixa metal-free. Paciente do gênero masculino, 38 anos, procurou atendimento queixando-se da estética do seu sorriso. No exame clínico foi observada uma coroa total metalocerâmica no elemento 21 e facetas de resina composta nos elementos 11,12 e 22 , todas com estética insatisfatória. O dente 21 apresentava um núcleo metálico fundido adequado e os dentes 12 e 22 com tratamentos endodônticos. Foi proposta a instalação de pinos de fibra de vidro nos dentes 12 e 22 . Além, da confecção de coroas totais metais-free em dissilicato de lítio nos quatro incisivos superiores com o sistema e-max ceram. O resultado final estético e funcional foi aprovado pelo paciente e pelos profissionais envolvidos.

Descritores: Estética Dentária; Cerâmica; Reabilitação Bucal.

\begin{abstract}
To achieve aesthetics in anterior teeth one must be aware of several techniques and especially make the correct choice of materials. Dental ceramics are increasingly present in restorations, since they have excellent optical and mechanical properties. Thus, the objective of this study was to achieve esthetics in anterior teeth with different substrate through a fixed prosthesis metal-free. A male patient, 38 years old, sought care complaining about the aesthetics of his smile. In the clinical examination a total metaloceramic crown was observed in element 21 and composite resin facets in elements 11,12 and 22, all with unsatisfactory aesthetics. Tooth 21 had a suitable molten metal core and teeth 12 and 22 with endodontic treatments. It was proposed to install fiberglass pins on the teeth 12 and 22 . In addition, from the manufacture of total metal-free crowns in lithium disilicate in the four upper incisors with the e-max system ceram. The final aesthetic and functional result was approved by the patient and the professionals involved.
\end{abstract}

Descriptors: Esthetics, Dental; Ceramics; Mouth Rehabilitation.

\section{Resumen}

Para alcanzar la estética en dientes anteriores se debe tener conocimiento de diversas técnicas y principalmente hacer la elección correcta de los materiales. Las cerámicas dentales están cada vez más presentes en las restauraciones, ya que poseen óptimas propiedades ópticas y mecánicas. Así, el objetivo de este estudio fue proporcionar la estética en dientes anteriores con diferentes sustratos a través de una prótesis fija metal-free. Paciente del género masculino, 38 años, buscó atención quejándose de la estética de su sonrisa. En el examen clínico se observó una corona total metalocerámica en el elemento 21 y facetas de resina compuesta en los elementos 11,12 y 22 , todas con estética insatisfactoria. El diente 21 presentaba un núcleo metálico fundido adecuado y los dientes 12 y 22 con tratamientos endodónticos. Se propuso la instalación de pines de fibra de vidrio en los dientes 12 y 22. Además, de la confección de coronas totales metales libres en disilicato de litio en los cuatro incisivos superiores con el sistema e-max ceram. El resultado final estético y funcional fue aprobado por el paciente y los profesionales involucrados.

Descriptores: Estética; Cerámica; Rehabilitación Bucal.

\section{INTRODUÇÃO}

A estética na Odontologia se baseia em regras, leis e técnicas, além de princípios lógicos para desenvolver um sorriso harmônico. Esses princípios se baseiam em reproduzir dentes com proporções corretas e que consigam estabelecer um equilíbrio com os tecidos gengivais. Atualmente, a aparência estética dental reflete uma grande importância na aceitação e autoestima das pessoas. Assim, a busca por tratamentos estéticos na odontologia impulsionam a procura por alternativas de tratamentos que proporcionem uma estética adequada ${ }^{1}$.

As resinas compostas são os materiais mais utilizados para restaurações estéticas, porém simbolizam um grande desafio na prática odontológica $^{2}$. Apresentam fraturas frequentes $\mathrm{e}$ ocorrência de cárie secundaria ${ }^{3}$. Além disso, pode ocorrer descoloração e, consequentemente, necessidade de troca periódica pelos clínicos ${ }^{4}$.

As cerâmicas odontológicas vêm se consagrando na Odontologia estética, por apresentarem propriedades semelhantes às dos dentes naturais, como estabilidade de cor, biocompatibilidade, elevada resistência à compressão e coeficiente de expansão térmica semelhante à estrutura dentária ${ }^{5}$. Ainda, com o acréscimo de cristais de dissilicato de lítio na formulação das cerâmicas feldspáticas, houve um favorecimento nas propriedades mecânicas, sem comprometer suas propriedades ópticas, por apresentar índice de refração da luz semelhante ao do esmalte dental, mimetizando o aspecto natural dos dentes ${ }^{6}$.

A combinação de cores das restaurações com os dentes naturais é um dos aspectos mais 
desafiadores de uma reabilitação estética anterior, visto que existe uma alta complexidade em reproduzir as características ópticas dos dentes. Dessa forma, a necessidade de obter restaurações semelhantes aos dentes naturais torna-se um dos grandes desafios da Odontologia ${ }^{7}$. Visando um resultado estético satisfatório, o objetivo desse estudo foi descrever os procedimentos de um tratamento com o desafio de se conseguir um sorriso estético através de prótese fixa metal-free.

\section{CASO CLÍNICO}

O paciente RTS, 38 anos, gênero masculino, compareceu a clínica de Prótese Parcial Fixa da Faculdade de Odontologia de Araçatuba FOA/UNESP queixando-se da estética do seu sorriso (Figura 1).

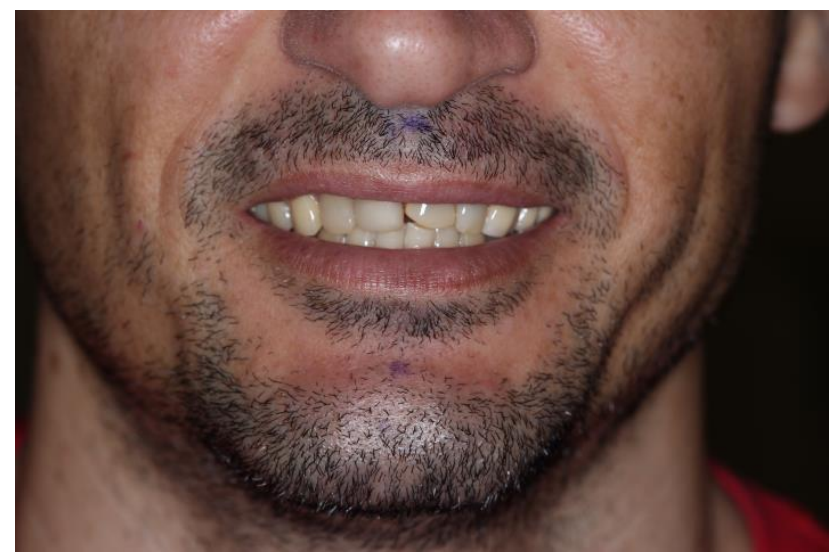

Figura 1: Sorriso antiestético do paciente.

Foi realizado exame clínico, no qual foi verificada a presença de uma coroa total metalocerâmica no elemento 21 e facetas de resina composta nos dentes 11, 12 e 22, apresentando estética insatisfatória, devido à aspectos de opacidade, formatos e contornos inapropriados (Figura 2).

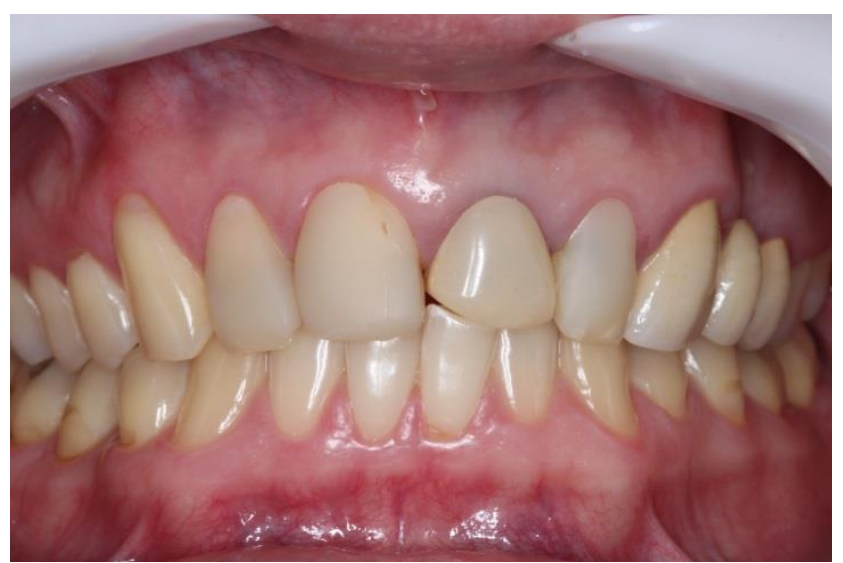

Figura 2: Situação inicial do sorriso do paciente, apresentando aspectos inapropriados.

No exame radiográfico, foi verificado que os elementos 12 e 22 apresentavam tratamento endodôntico satisfatório e o elemento 21 apresentava um núcleo metálico fundido satisfatório.
Após a obtenção dos modelos de estudo com hidrocolóide irreversível (Hydrogum, Zhermack), os modelos foram montados em articulador semiajustável (Bio-Art) e foi proposta a confecção de coroas totais livres de metal nos quatro incisivos superiores com o sistema e.max Ceram (Ivoclar Vivadent). Na primeira etapa foi realizada a desobturação de $2 / 3$ do tratamento endodôntico dos elementos 12 e 22 com brocas largo (Peeso AR, Microdont) para instalação de pinos de fibra de vidro (Whitepost, FGM) número 2 (Figura 3).

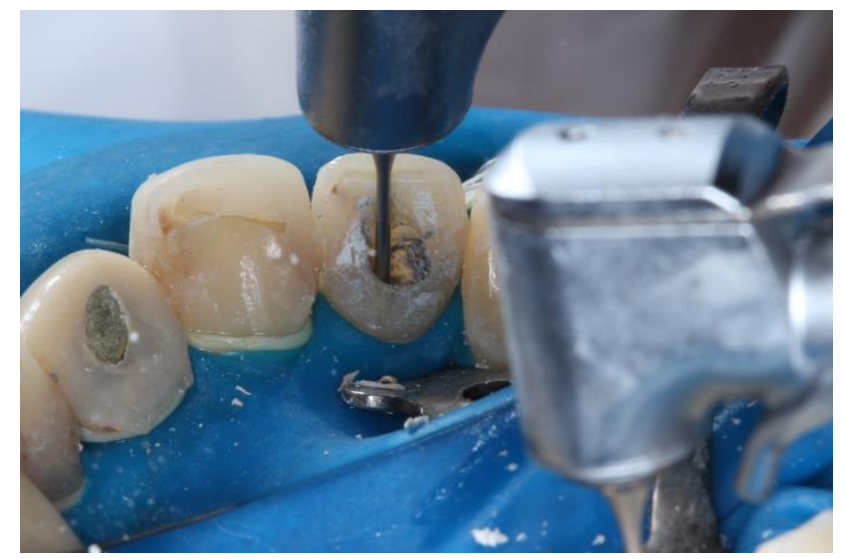

Figura 3: Desobturação do elemento 22.

Os pinos de fibra de vidro foram armazenados em recipiente com álcool absoluto por 5 minutos, aplicado ácido fosfórico 37\% (Condor, FGM) para remoção de detritos, lavados em água corrente e, posteriormente, aplicada o silano (Monobond, Ivoclair Vivadent) (Figura 4).

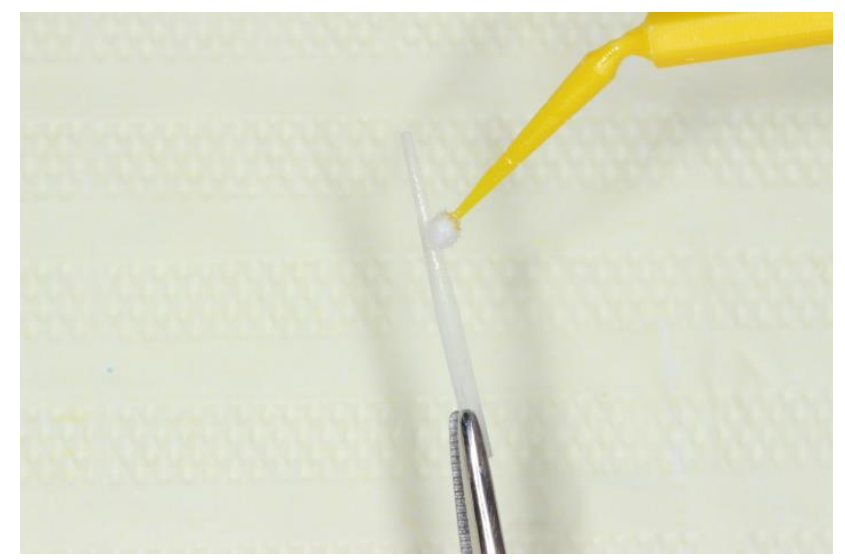

Figura 4: Silanização do pino de fibra de vidro.

Foram cimentados nos elementos com cimento resinoso autoadesivo (Relyx U-200, 3M). Posteriormente, foi realizado aplicação de ácido fosfórico 37\% e adesivo (Single Bond 2, 3M) nos pinos e remanescente coronário para reconstrução com resina composta $(Z 350,3 \mathrm{M})$ e fotopolimerização (PolyWireless, Kavo) (Figura 5).

Previamente, foi solicitado ao laboratório de prótese a confecção de coroas provisórias em resina acrílica termopolimerizável (Clássico) para os quatro elementos. Para a realização dos preparos para coroa 
total, foi removida a coroa metalocerâmica do dente 21 utilizando uma ponta diamantada \#3145 (KG Sorensen) para remover a cerâmica de cobertura e uma broca trans-metal (Microdont) para remover o coping metálico (Figura 6).

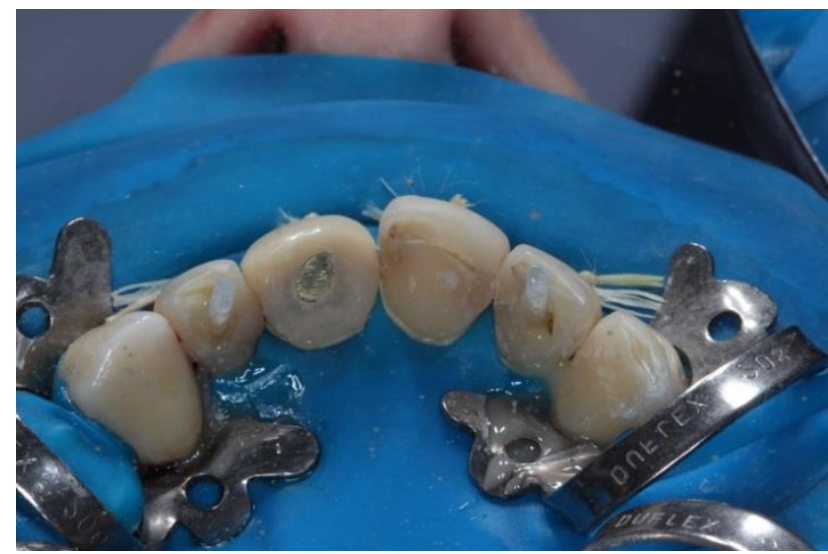

Figura 5: Situação após instalação dos pinos de fibra de vidro.

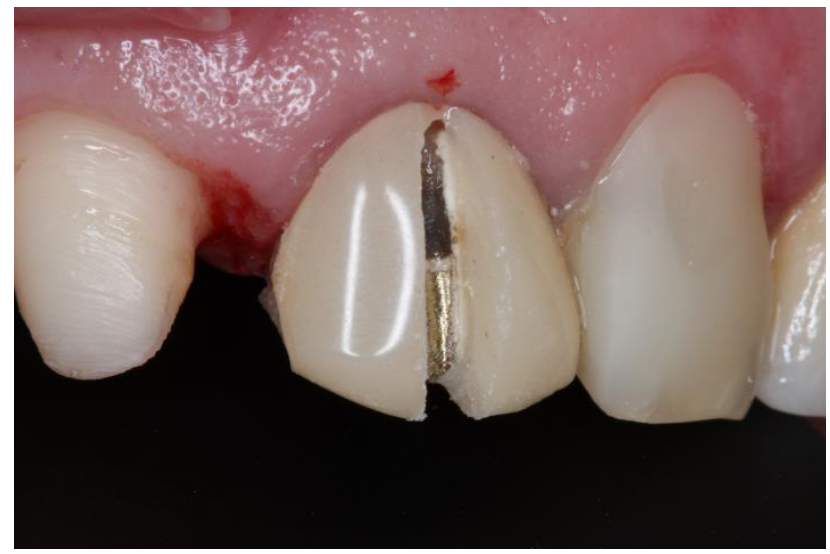

Figura 6: Remoção da coroa metalocerâmica do elemento 21.

Assim, foi realizado o preparo do tipo chanfro largo com a ponta diamantada \#3228 (KG Sorensen) nos quatro elementos (Figura 7).

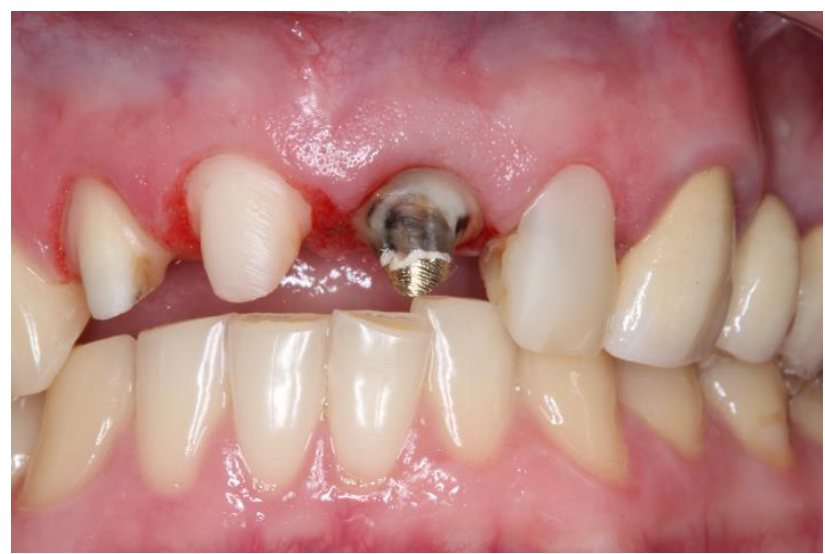

Figura 7: Realização dos preparos dentais.

Após os preparos realizados, os provisórios foram reembasados e, antes da cimentação, foi realizada profilaxia dos preparos com pedra pomes (SS White) e água com taça de borracha (Microdont). Os elementos foram cimentados com o cimento provório (Temp-Bond, Kerr) realizando a remoção dos excessos e ajuste oclusal (Figura 8).

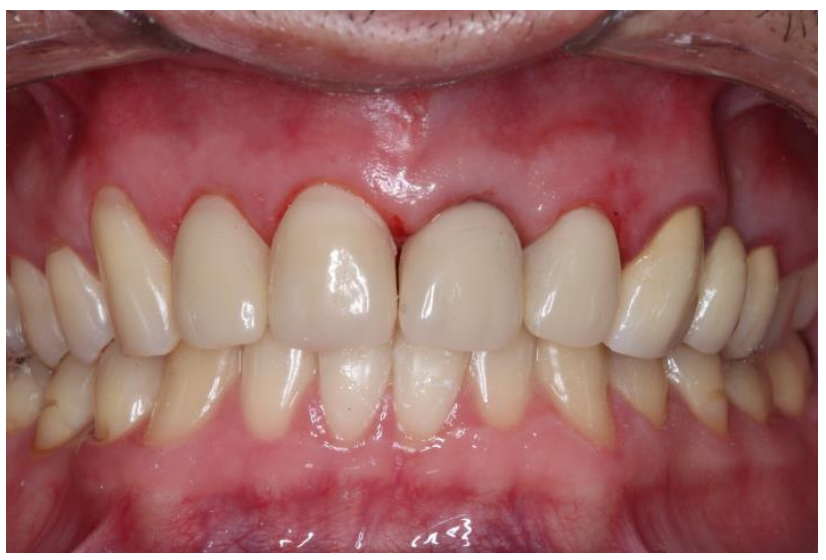

Figura 8: Situação após cimentação das coroas provisórias.

Na moldagem para confecção dos copings foi utilizada a técnica da dupla impressão ou reembasamento com duplo fio. Inicialmente, a arcada superior foi moldada com o silicone de adição pesado (Express XT Denso, 3M ESPE) em uma moldeira de estoque perfurada (Tecnodent) (Figura 9).

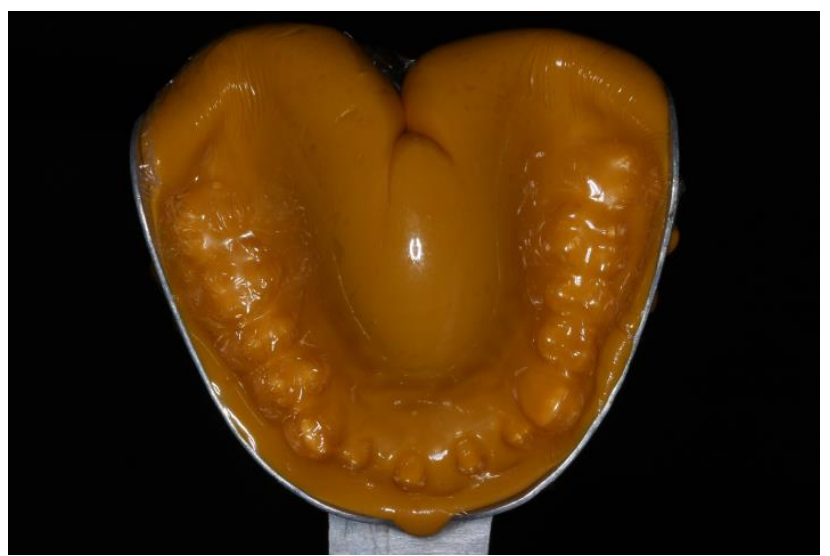

Figura 9: Moldagem com silicone de adição pesado em moldeira perfurada.

O afastamento gengival foi realizado, inicialmente, com o fio retrator \#00 (Ultrapak, Vivadent) embebido em solução hemostática (Hemostop, Dentsply), seguido do fio \#0. O fio retrator \#0 foi removido cuidadosamente e a pasta leve (Express XT Light Body, 3M ESPE) do silicone de adição foi levado em todos os preparos (Figura $10)$.

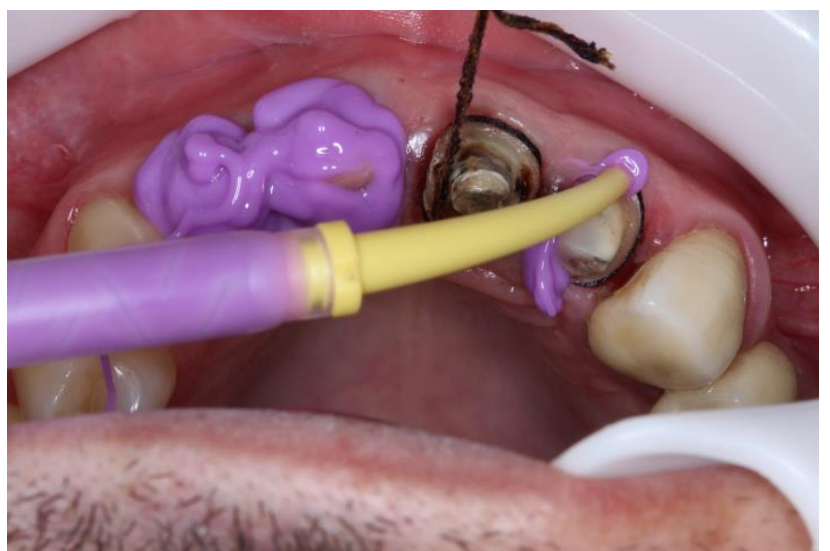

Figura 10: Silicone de adição sendo levado em todos os preparos. 
Assim, o molde inicalmente obtido com o silicone pesado foi levado em posição sobre o leve e aguardado o tempo de presa do material (Figura 11).

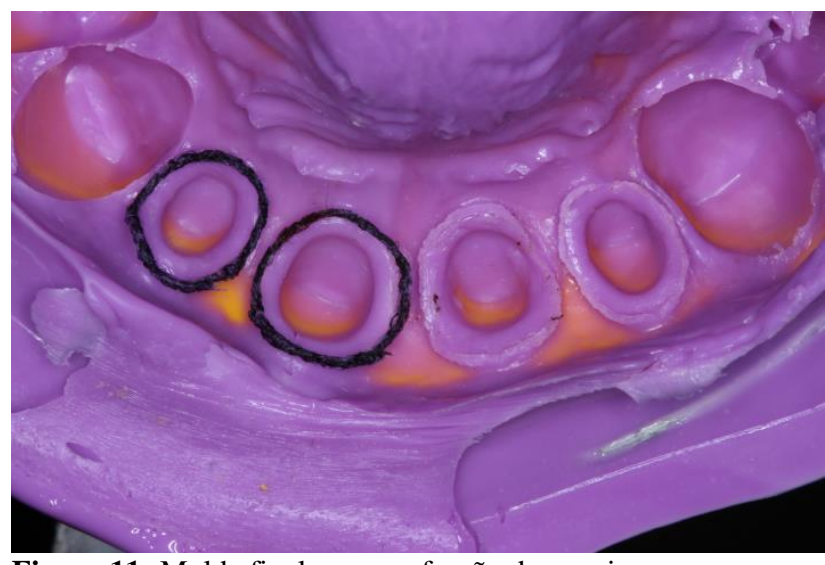

Figura 11: Molde final para confecção dos copings.

Os copings foram confeccionados em dissilicato de lítio (HT -12, 11, 22 e LT - 21) (Figura 12) e provados sobre os preparos (Figura 13).

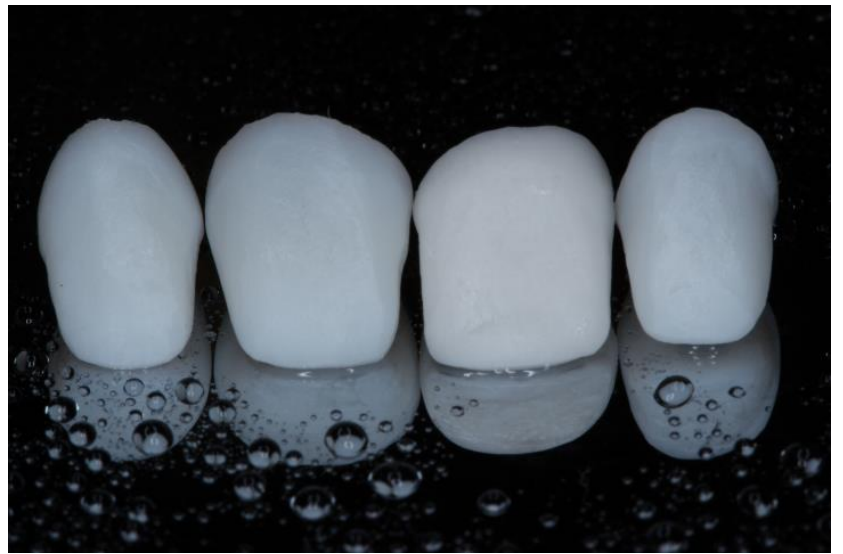

Figura 12: Copings confeccionados em dissilicato de lítio.

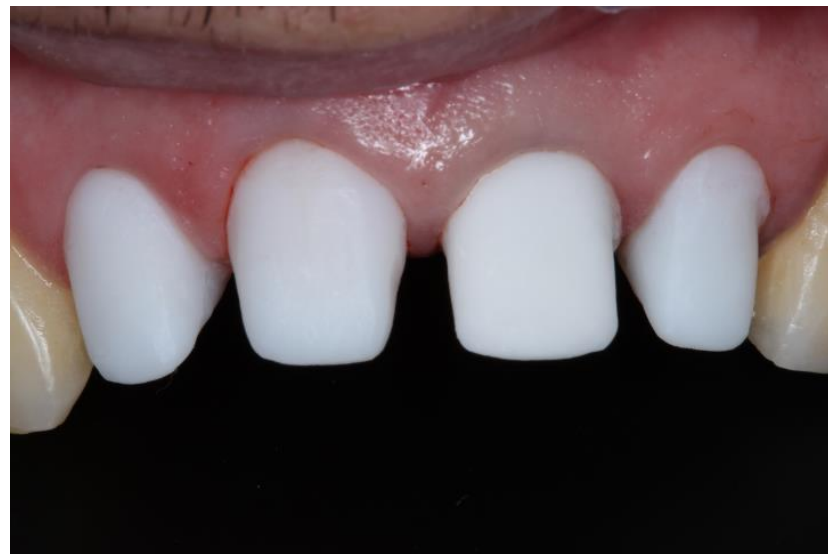

Figura 13: Copings sendo provados nos elementos.

Foi realizada a prova dos copings e feito o registro de posição dos mesmos (Figura 14). A moldagem de transferência foi realizada com silicone de adição (Express XT Denso, 3M ESPE) leve e pesado (Figura 15). Então, o molde foi encaminhado para o laboratório para aplicação da cerâmica de cobertura (Figura 16). Após a confecção, as peças foram posicionadas sobre os preparos e realizadas os testes com o sistema de cimentos Relyx Try-in (3M ESPE), a fim de verificar a cor do cimento definitivo. Também foi realizado pré-ajuste oclusal com papel carbono (Super Carbon, Maquira).

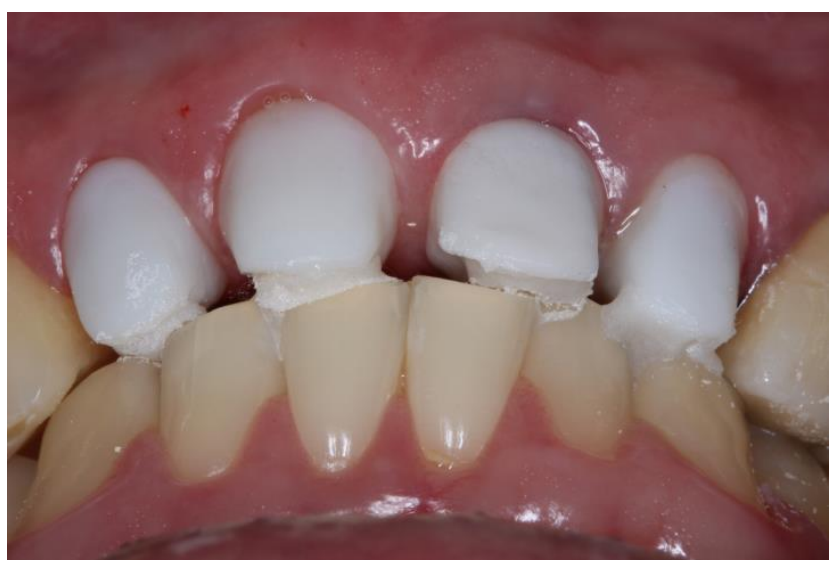

Figura 14: Registro de posição dos copings.

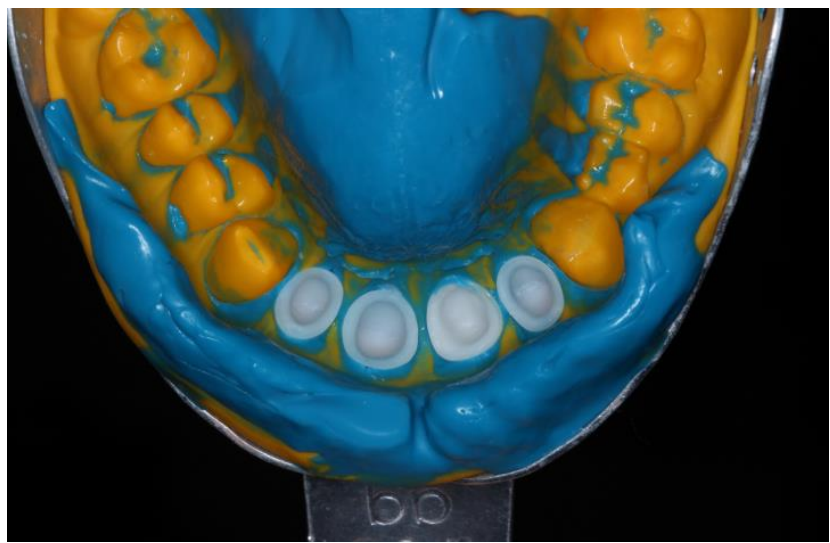

Figura 15: Moldagem de transferência dos copings.

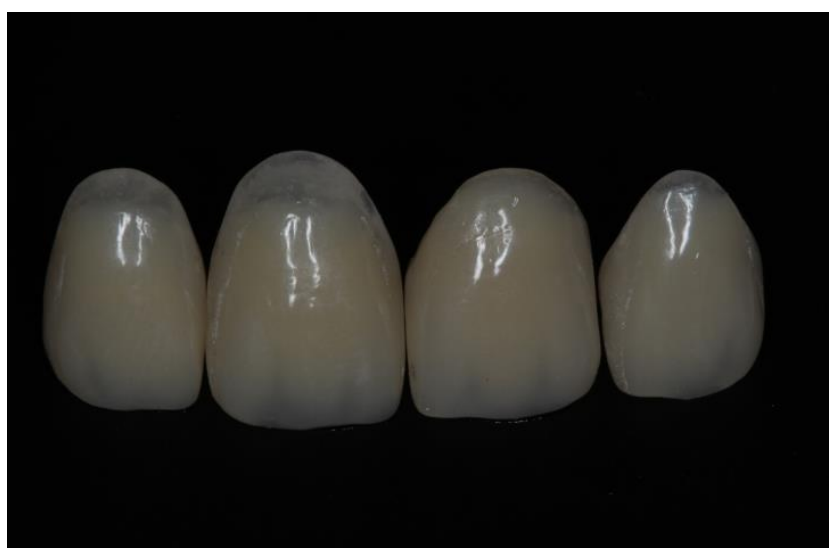

Figura 16: Aplicação da cerâmica de cobertura.

Após a escolha da cor do cimento (translúcido), foi realizada a preparação das peças cerâmicas, para cimentação, com o condicionamento das peças com ácido fluorídrico (Porcelain Etchant 9,5\%, Bisco Inc.) por 20 segundos (Figura 17). Foram lavadas com água, secas com jatos de ar e condicionadas com ácido fosfórico $37 \%$ por 60 segundos (Figura 18), lavadas e secas novamente. Foi realizada a silanização com o agente de união (Monobond Plus, Ivoclair Vivadent) (Figura 19).

Após o preparo das peças, foi iniciada a preparação dos dentes. Estes foram isolados, 
realizado profilaxia com pedra pomes e água. Foi realizado o condicionamento com ácido fosfórico a $37 \%$ durante 15 segundos (Figura 20), lavados e secos.

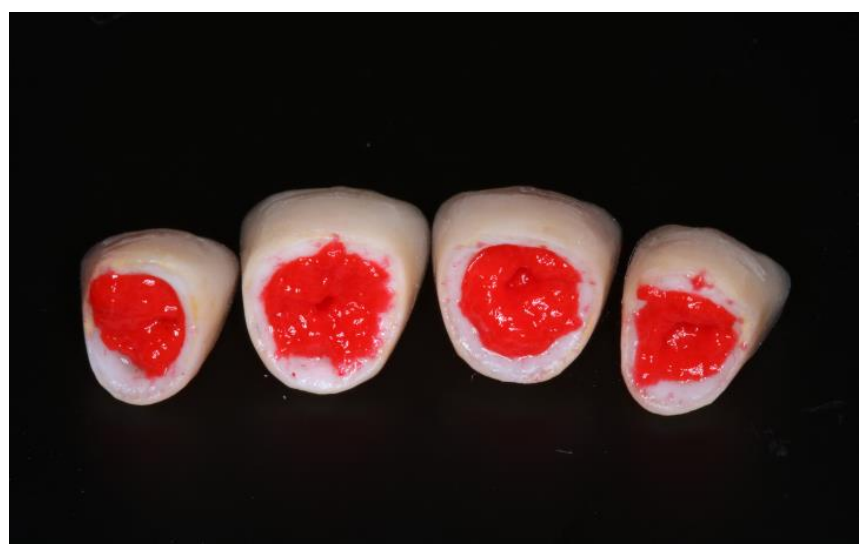

Figura 17: Condicionamento das peças com ácido fluorídrico.

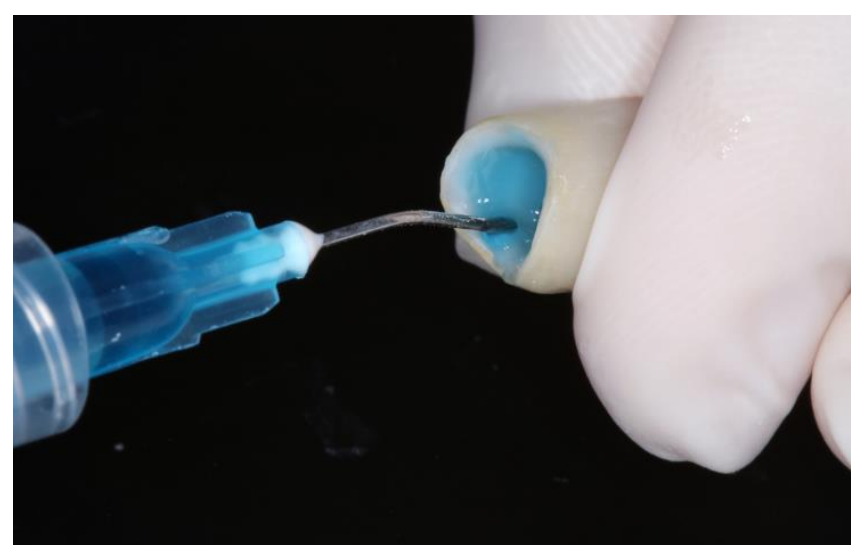

Figura 18: Condicionamento com ácido fosfórico.

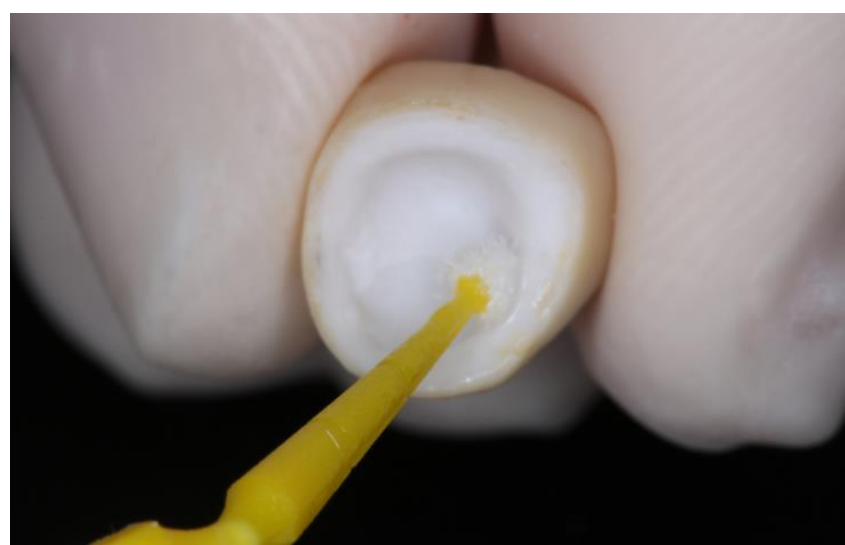

Figura 19: Silanização das peças.

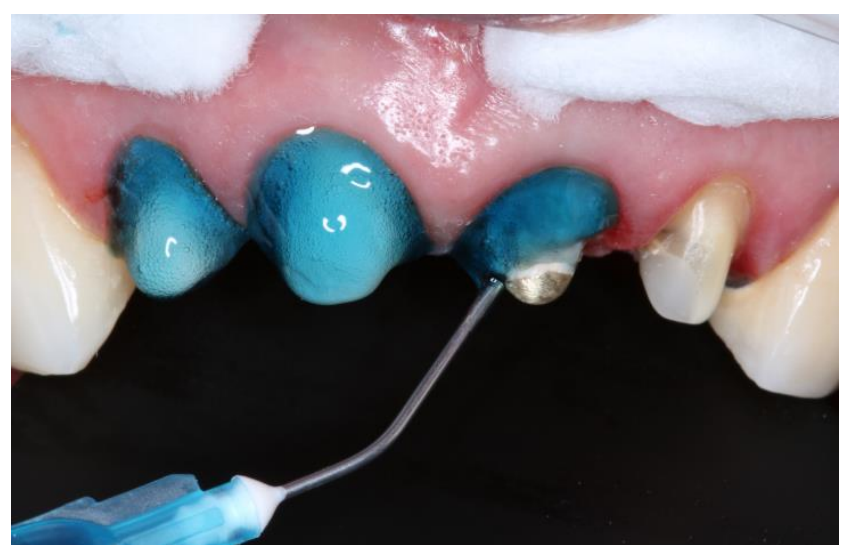

Figura 20: Condicionamento dos elementos dentais com ácido fosfórico.

Em seguida, foi aplicado o adesivo (Excite $\mathrm{F}$ DSC, Ivoclar Vivadent) (Figura 21), o qual foi fotopolimerizado por 40 segundos. Foi utilizado o cimento resinoso dual (Variolink II, Ivoclair Vivadent), o qual foi removido os excessos e fotopolimerizado por 20 segundos em cada face (Figuras 22 e 23).

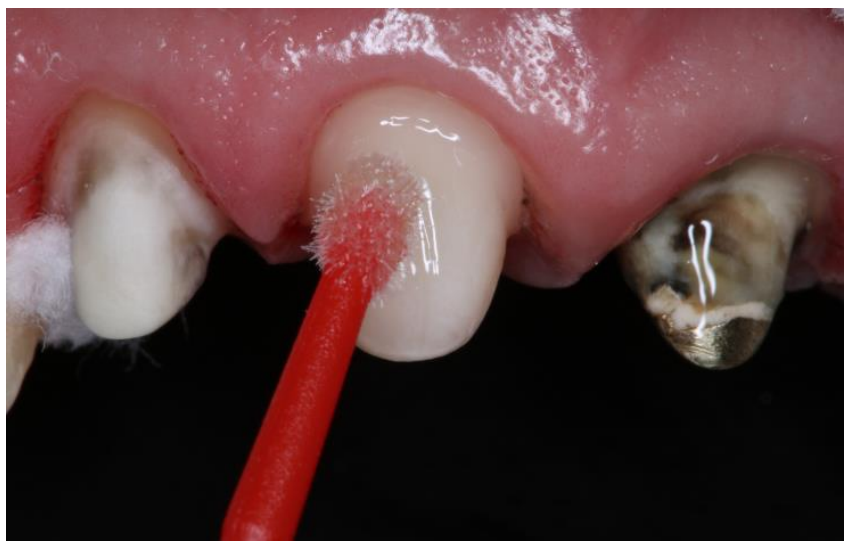

Figura 21: Aplicação do sistema adesivo.

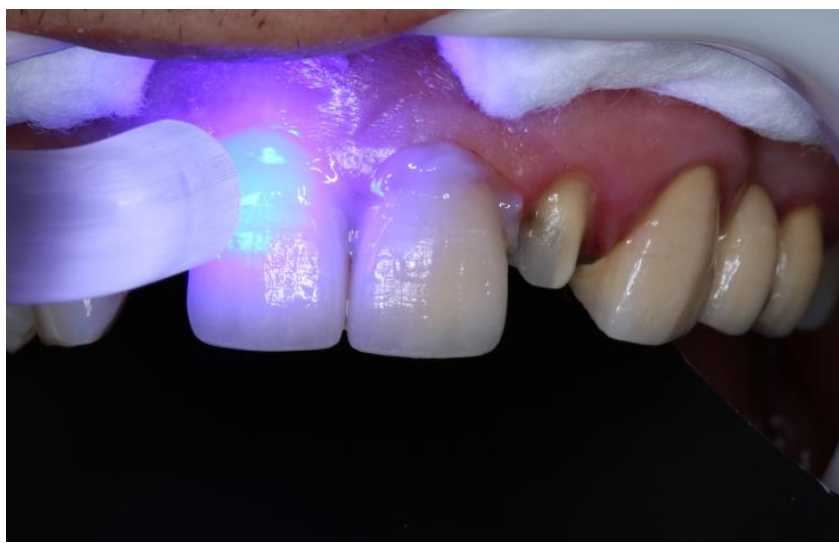

Figura 22: Fotopolimerização do cimento resinoso.

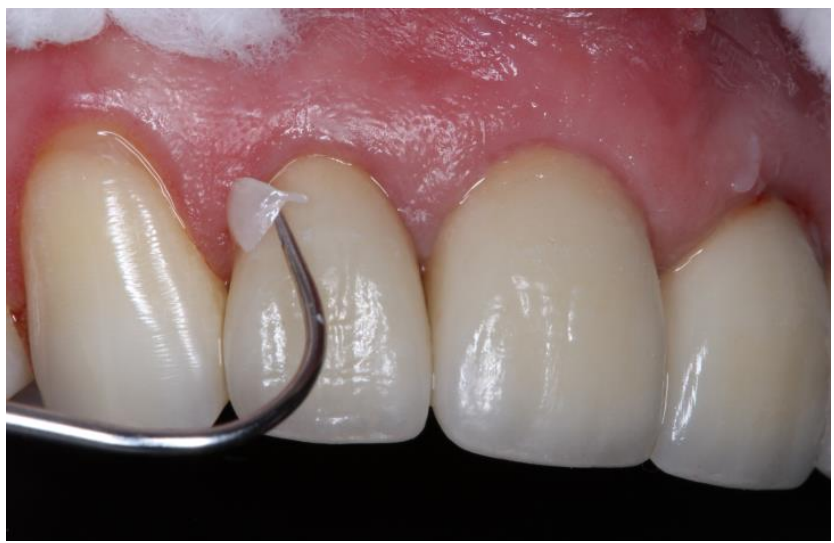

Figura 23: Remoção do excesso de cimento dos elementos.

$\mathrm{Na}$ sequência, foi realizado o ajuste oclusal de acordo com as guias anteriores e caninas do paciente. $\mathrm{O}$ resultado final estético e funcional foi aprovado pelo paciente e pelos profissionais envolvidos (Figuras 24 e 25). 


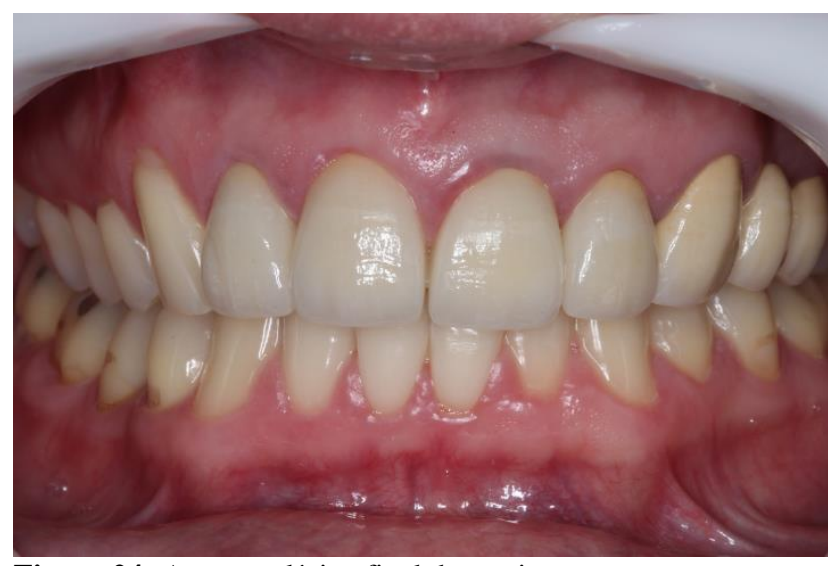

Figura 24: Aspecto clínico final do sorriso.

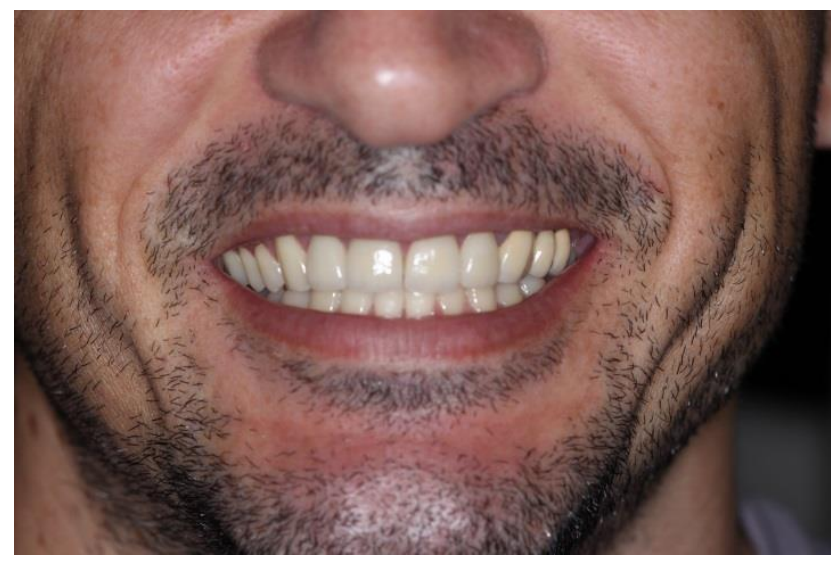

Figura 25: Resultado final do sorriso do paciente.

\section{DISCUSSÃO}

Atualmente, tornou-se possível a confecção de próteses fixas totalmente cerâmicas, sem necessidade de reforço metálico. O que resulta em uma estética mais satisfatória, devido à ausência do escurecimento da margem gengival, que pode ocorrer com o passar do tempo em próteses metalocerâmicas ${ }^{8}$.

Existem diferentes tipos de cerâmicas utilizadas na odontologia, com diferentes graus de translucidez, as quais podem ser alteradas pela estrutura, espessura, composição cristalina, porosidade entre as camadas e reflexo da infraestrutura entre a interface e a cobertura de cerâmica ${ }^{9}$. Através das qualidades obtidas pela cerâmica reforçada por dissilicato de lítio, como resistência mecânica e propriedades ópticas semelhantes às dos dentes naturais ${ }^{4}$ se tornou $o$ material de escolha para a realização do presente caso, visto a exigência estética e funcional da reabilitação dos dentes anteriores, devendo ser minuciosamente analisada e reproduzida.

A utilização de retentores intrarradiculares tem como finalidade proporcionar condições adequadas para que uma reabilitação seja executada corretamente e, assim, evitando futuros problemas para as funções mastigatórias ${ }^{10}$. Os retentores intrarradiculares são divididos em núcleos metálicos fundidos e os pinos pré-fabricados ${ }^{11}$. Um dos pinos pré-fabricado mais utilizado é o de fibra de vidro, pois apresenta translucidez, o que aumenta o grau de estética do resultado final e boa adesão aos compósitos dentais ${ }^{12}$ contribuindo para uma adequada restauração dos elementos 12 e 22. Como os elementos 12,11 e 22 possuíam um substrato natural, logo foi possível utilizar o dissilicato de lítio com alta translucidez, o que permite uma maior passagem de luz através do material e uma aparência mais realista dos dentes ${ }^{13}$. Já com o elemento 21 , por conter um núcleo metálico fundido, foi utilizado o dissilicato de lítio com baixa translucidez para conseguir ocultar seu substrato escurecido.

O sucesso de restaurações em cerâmica pura também depende da ligação entre tecidos duros cerâmicos e dentais por materiais de cimentação. Nos últimos anos, os cimentos resinosos se destacaram devido a sua baixa solubilidade, boa estética, uma vez que promove estabilidade cromática e propriedades mecânicas adequadas ${ }^{14,15}$. No caso descrito, a escolha foi para a utilização de cimento resinoso dual, visto que um cimento resinoso apenas fotopolimerizável não conseguiria alcançar os níveis adequado para uma correta polimerização, devido as peças oferecem uma espessura de cerâmica maior que $1 \mathrm{~mm}^{16}$. O cimento resinoso também atua como um fator a ser considerado quando a estética é essencial para a conclusão do caso ${ }^{17}$. Desta forma, o uso de pastas try-in são essenciais, já que oferecem tanto para o clínico, como para o paciente uma previsão de como as peças ficarão após a cimentação final ${ }^{18}$.

Os materiais são um dos principais fatores para um bom desempenho clínico. Assim, restaurações cerâmicas metal-free se tornam uma boa opção para a substituição de restaurações metalocerâmicas, visto que possuem uma boa biomecânica e uma melhor estética ${ }^{19}$.

\section{CONCLUSÃO}

Diante do caso clínico, podemos constatar que há um grande desafio em estabeler uma estética em dentes anteriores, principalmente, quando há núcleo metálico fundido envolvido. Porém com materiais bem indicados, como a cerâmica de dissilicato de lítio e cimentos resinosos foi possível alcançar um resultado satisfatório.

\section{REFERÊNCIAS}

1. Okida RC, De Moura AP, Franco LM, Salomão FM, Rahal S, Machado LS, Okida DSS. A utilização do DSD (Digital Smile Design) para a otimização da estética dental. Rev Odontol Araçatuba 2017;38(3):9-14.

2. Sabatini C. Color stability behavior of methacrylate-based resin composites polymerized with light-emitting diodes and quartz-tungstenhalogen. Oper Dent. 2015;40(3):71-81.

3. Wang X, Huyang G, Palagummi SV, Liu X, Skrtic D, Beauchamp C et al. High performance 
dental resin composites withhydrolytically stable monomers. Dent Mater. 2018;34(2):228-37.

4. Yazici AR, Celik C, Dayangaç B, Ozgünaltay G. The effect of curing units and staining solutions on the color stability of resin composites. Oper Dent. 2007; 32(6):616-22.

5. Aguiar EMG, Rodrigues RB, Lopes CCA, Silveira Júnior CD, Soares CJ, Novais VR. Diferentes sistemas cerâmicos na reabilitação oral: relato de caso clínico. Rev Odontol Bras Central. 2016;25(72):31-6.

6. Mazaro JVQ, Zavanelli AC, Pellizzer EP, Verri FR, Falcón-Antennucci RM. Considerações clínicas para a restauração da região anterior com facetas laminadas. Rev Odontol Araçatuba. 2009;30(1):51-4.

7. Vichi A, Louca C, Corciolani G, Ferrari M. Color related to ceramic and zirconia restorations: a review. Dent Mater. 2011;27(1):97-108.

8. Rossato DM, Saade EG, Saad JRC, Porto-Neto ST. Coroas estéticas anteriores em cerâmica metal-free: relato de caso clínico. Rev Sul-Bras Odontol. 2010;7(4):494-98.

9. Lanza MDS, Andreeta MRB, Pegoraro TA, Pegoraro LF, Carvalho RM. Influence of curing protocol and ceramic composition on the degree of conversion of resin cement. J Appl Oral Sci. 2017;25(6):700-7.

10. Soares PFB, Siqueira JM, Carvalho VF, Bicalho AA, Soares CJ. Contenção interdentária empregando fibra de vidro e resina composta: Relato de caso e acompanhamento de 14 anos. Rev Odontol Bras Central. 2016;25(73):80-3.

11. Martinez-Insua A, da Silva L, Rilo B, Santana U. Comparison of the fracture resistances of pulpless teeth restored with a cast post and core or carbonfiber post with a composite core. J Prosthet Dent. 1998;80(5):527-32.

12.Rocha IJPB, Silva LDR, de Santa Maria SL, Oliveira DP, Porfírio Z. Análise de dois métodos de desinfecção de condutos radiculares após preparo para pinos: proposta de protocolo protético: estudo in vitro. Rev Odontol UNESP. 2017;46(4):189-95.

13.Dede DÖ, Ceylan G, Yilmaz B. Effect of brand and shade of resin cements on the final color of lithium disilicate ceramic. J Prosthet Dent. 2017;117(4):539-44.

14.Lopes Cde C, Rodrigues RB, Silva AL, Simamoto Júnior PC, Soares CJ, Novais VR. Degree of conversion and mechanical properties of resin cements cured through different all-ceramic systems. Braz Dent J. 2015;26(5):484-89.

15. Martins FV, Vasques WF, Fonseca EM. How the variations of the thickness in ceramic restorations of lithium disilicate and the use of different photopolymerizers influence the degree of conversion of the resin cements: a systematic review and meta-analysis. J Prosthodont. 2019;28(1):e395-403.

16.Turp V, Turkoglu P, Sen D. Influence of monolithic lithium disilicate and zirconia thickness on polymerization efficiency of dualcure resin cements. J Esthet Restor Dent. 2018;30(4):360-68.

17.Tavarez RR, Gonçalves LM, Dias AP, Dias AC, Malheiros AS, Silva AC. An harmonic smile resulted from the use of ceramic prosthesis with zirconia structure: a case report. J Int Oral Health. 2014;6(3):90-2.

18.Vaz EC, Vaz MM, de Torres ÉM, de Souza JB, Barata TDJE, Lopes LG. Resin cement: correspondence with try in paste and influence on the immediate final color of veneers. J Prosthodont. 2019;28(1):e74-81.

19.Ladha K, Verma M. Conventional and contemporary luting cements: an overview. J Indian Prosthodont Soc. 2010;10(2):79-88.

\section{CONFLITO DE INTERESSES}

Os autores declaram não haver conflitos de interesse.

\section{AUTOR PARA CORRESPONDENCIA}

\section{Aldieris Alves Pesqueira}

aldieris.pesqueira@unesp.br

Submetido em 13/09/2018

Aceito em 17/01/2019 